\title{
The 4-m International Liquid Mirror Telescope
}

Jean Surdej ${ }^{1 *}$, Paul Hickson ${ }^{2}$, Ermanno Borra ${ }^{3}$, Jean-Pierre Swings ${ }^{1}$, Serge Habraken ${ }^{1}$, Talat Akhunov ${ }^{4,5}$, Przemyslaw Bartczak ${ }^{6}$, Hum Chand ${ }^{7}$, Michaël De Becker ${ }^{1}$, Ludovic Delchambre ${ }^{1}$, François Finet ${ }^{8}$, Brajesh Kumar ${ }^{9}$, Anil Pandey ${ }^{7}$, Anna Pospieszalska ${ }^{1}$, Bikram Pradhan $^{1}$, Ram Sagar $^{9}$, Olivier Wertz ${ }^{10}$, Peter De Cat ${ }^{11}$, Stefan Denis ${ }^{12}$, Jonathan de Ville ${ }^{12}$, Mukesh Kumar Jaiswar ${ }^{7}$, Patricia Lampens ${ }^{11}$, Nandish Nanjappa ${ }^{7}$, Jean-Marc Tortolani ${ }^{12}$

${ }^{1}$ Space sciences, Technologies and Astrophysics Research (STAR) Institute,

Université de Liège, Allée du 6 Août 19c, 4000 Liège, Belgium

${ }^{2}$ Department of Physics and Astronomy, The University of British Columbia, 6224 Agricultural Road, Vancouver, BC, V6T1Z1, Canada

${ }^{3}$ Department of Physics, Université Laval, 2325, rue de l'Université, Québec (Québec) G1V 0A6, Canada

${ }^{4}$ National University of Uzbekistan, Physics Faculty, Department of Astronomy and Atmospheric physics, Tashkent, 100174, Uzbekistan

${ }^{5}$ Ulugh Beg Astronomical Institute of the Uzbek Academy of Sciences, Astronomicheskaya 33, 100052, Tashkent, Uzbekistan

${ }^{6}$ Poznań Observatory, Adam Mickiewicz University, Sloneczna 36, 60-101 Poznań, Poland

${ }^{7}$ Aryabhatta Research Institute of observational sciencES (ARIES), Manora Peak Nainital-263002, Uttarakhand (India)

${ }^{8}$ National Astronomical Observatory of Japan (NAOJ), 650 N. A'ohoku Place, Hilo, 96720 HI, USA

${ }^{9}$ Indian Institute of Astrophysics, Koramangala, Bangalore 560 034, India

${ }^{10}$ Argelander-Institut für Astronomie, Auf dem Hügel 71, D-53121 Bonn 9, Germany

${ }^{11}$ Royal Observatory of Belgium, Avenue Circulaire 3, 1180 Brussels, Belgium

${ }^{12}$ Advanced Mechanical and Optical Systems (AMOS), Liège Science Park, rue des Chasseurs Ardennais 2, 4031 Angleur, Belgium

*Also, hon. Research Director at F.R.S.-FNRS, Belgium; jsurdej@ulg.ac.be 
Abstract: The 4-m International Liquid Mirror Telescope (ILMT) is presently (March-June 2017) being erected on the ARIES site in Devasthal (Uttarakhand). We describe and illustrate in the present paper its different components. The ILMT will be used in the Time Delayed Integration (TDI) mode to carry out a deep survey and high S/N photometric and astrometric observations of solar system, galactic and extra-galactic objects within a narrow (24') strip of sky. In principle, the ILMT should detect and regularly monitor more than 50 multiply imaged quasars. It will also detect numerous supernovae (see Kumar et al., these proceedings) as well as space debris (see Pradhan et al., also in these proceedings).

\section{Introduction}

The 4-m International Liquid Mirror Telescope (ILMT) project results from a collaboration between the Institute of Astrophysics and Geophysics (Liège University), the Canadian Astronomical Institutes from Québec (Laval University), Montréal (University of Montreal), Toronto (University of Toronto and York University), Vancouver (University of British Columbia) and Victoria (University of Victoria) and the Aryabhatta Research Institute of Observational Sciences (ARIES, India). Several colleagues from the Royal Observatory of Belgium, the Poznań Observatory (Poland), the Ulugh Beg Astronomical Institute of the Uzbek Academy of Sciences and National University of Uzbekistan have also joined the project.

In 1850, Capocci (see Mailly 1872) has been the first to propose to use the surface of a liquid placed inside a cylindrical container that is in rotation around its vertical axis as a possible reflector. Indeed, under the constant pull of gravity and of the centrifugal acceleration, the surface of such a liquid takes the shape of a paraboloid. Given that a perfect reflecting paraboloid represents the ideal shape for a mirror to focus parallel light rays into a single point, a thin rotating layer of mercury will naturally focus the light from a distant star just above it, at its focal point, with the natural constraint that such a telescope always observes at the zenith.

The technology of liquid mirrors (LMs) has been developed by the team of Prof. Borra at Laval University (Borra et al. 1993). The first large fully operational LM Telescope has been the Large Zenithal Telescope (LZT) built by Prof. Hickson (Hickson et al. 2007, Hickson \& Racine 2007, Pfrommer, Hickson \& She 2009, Pfrommer and Hickson 2010, 2014).

The technology of LMs is relatively simple. Three components are required: i) a dish containing a reflecting liquid metal (essentially $\mathrm{Hg}$ ), ii) an air bearing on which the LM sits, and iii) a drive system. The mercury mirror of the ILMT has a 4 meter diameter with an aperture of $\mathrm{f} / 2$ defined by the speed of rotation. A $4 \mathrm{Kx} 4 \mathrm{~K}$ CCD camera manufactured by 'Spectral Instruments' and which can operate over the 4000 to $11000 \AA$ spectral range (SDSS filters $g^{\prime}, r^{\prime}, i^{\prime}$ are available), will be positioned at the prime focus of the ILMT at about 8 meters above the mirror. The mirror being parabolic in shape requires an optical corrector to get a flat focal surface of about 24' in diameter. All these elements are mechanically coupled by an external structure and a spider.

The rotation of the Earth induces the motion of the sky across the detector surface. The CCD detector works in the Time Delayed Integration (TDI) mode, i.e. it tracks the stars by electronically stepping the relevant charges at the same rate as the target drifts across the detector, allowing an integration as long as the target remains inside the detector area. The CCD detector is cooled down to a temperature near $-110^{\circ} \mathrm{C}$, in order to reduce as much as possible the dark current (see Figs. $1 \& 2$ ). The complete telescope is protected by a building with a sliding shutter for opening the roof. The building is air-conditioned and is also equipped with four exhaust fans. 


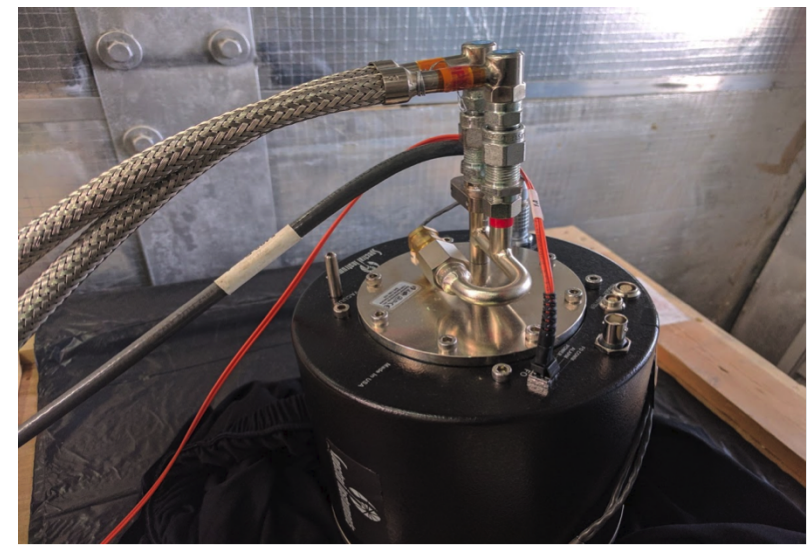

Figure 1: Spectral Instruments $4 \mathrm{Kx} 4 \mathrm{~K} \mathrm{CCD}$ camera : connections towards the compressor and the computer.

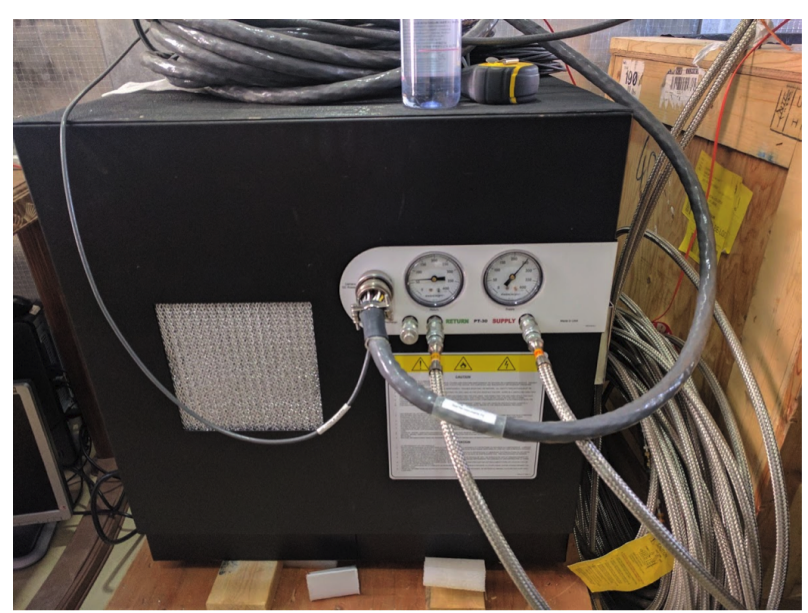

Figure 2: Compressor providing DC power and refrigerant supply to the CCD camera.

\section{Present status of the ILMT (June 2017)}

The ILMT equipment had been transported from Belgium to Devasthal in December 2011. However due to unexpected delays in the construction of the ILMT building, the erection of the telescope by AMOS (Advanced Mechanical and Optical Systems, Belgium) could only start in March 2017.

One compressor is needed to operate the air bearing. However, in order to avoid any interruption of the mirror rotation (cf. during the maintenance of the compressed air system), it is best to have two parallel air systems. Therefore we have decided to purchase two air compressors and two air tanks that will be working independently (see Fig. 3). If one air system fails or needs to be serviced, the second one will be automatically switched on. The two air compressors and air tanks have been installed on 27 February - 1 March 2017 by the Gardner-Denver company located in Delhi. In addition to the pneumatic air control system provided by AMOS (see Fig. 4), we have improved this by adding additional valves, air dryers, air filters and sensor (pressure, temperature, humidity and dew-point) modules along the two independent and parallel air systems to make sure that the air bearing will be operating under optimal and very safe conditions (see Figs. 5 \& 6).

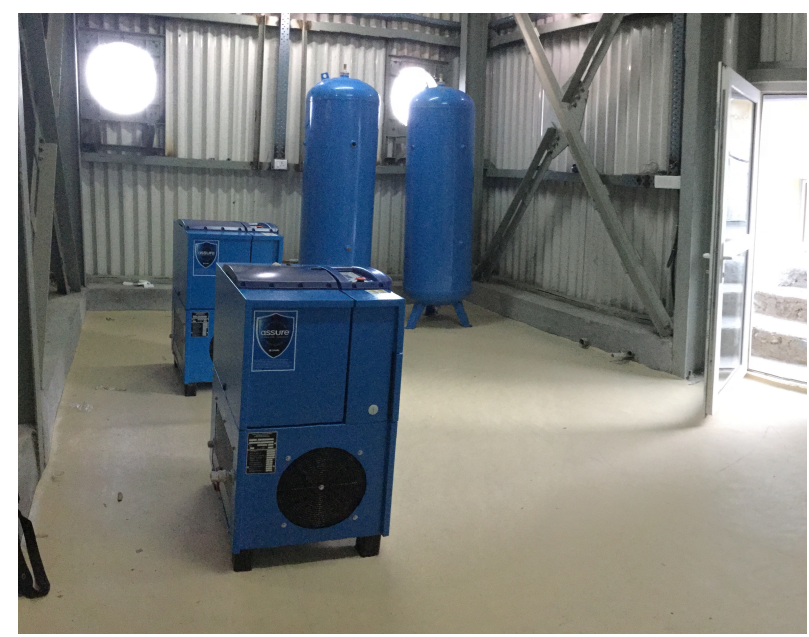

Figure 3: Compressor room with the two air compressors and air tanks.

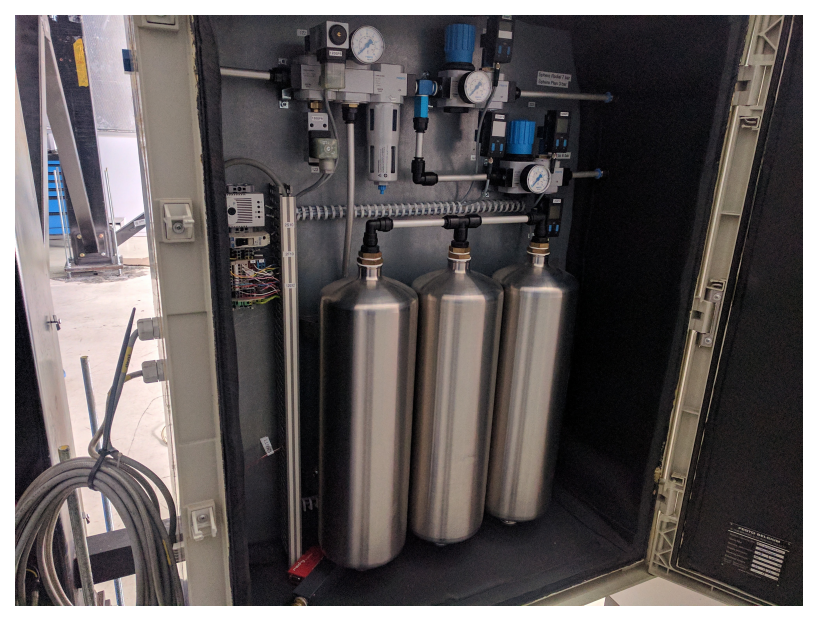

Figure 4: The AMOS pneumatic air control system installed in the ILMT main building. 


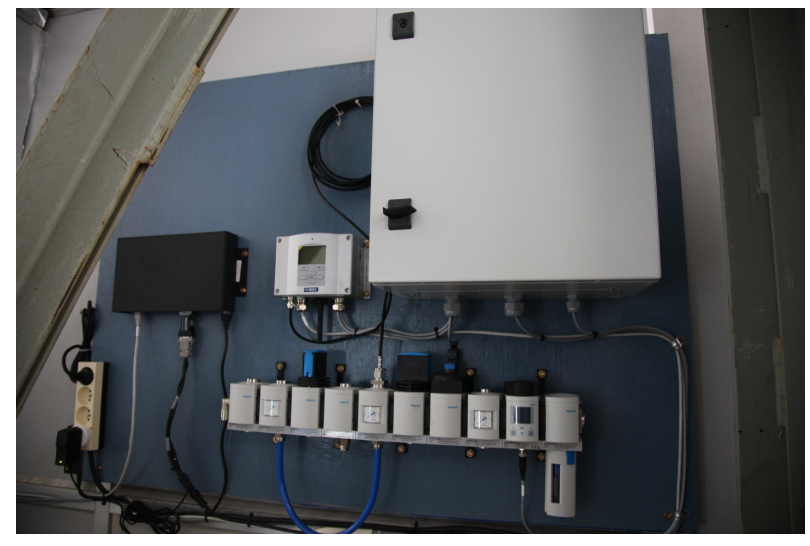

Figure 5: Improved pneumatic air control system installed in the ILMT control room.

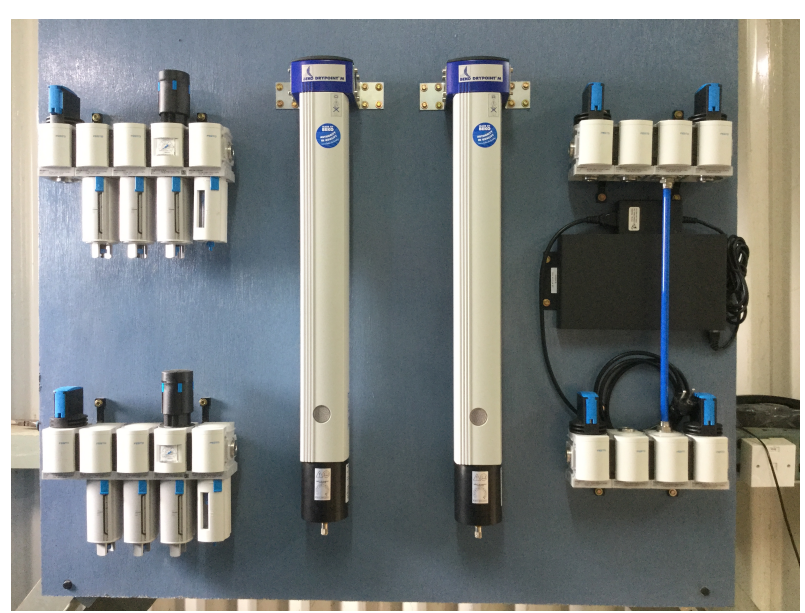

Figure 6: Improved pneumatic air control system installed in the compressor room.

On 2nd of March 2017, the ARIES and AMOS teams have proceeded with the transportation of the container in which the ILMT equipment had been stored in Devasthal since February 2012. One can see in Fig. 7 the ILMT equipment (telescope structure, air bearing, optical corrector) being unpacked. Some rust was found on various pieces of equipment but it has been successfully removed and cleaned by the AMOS team after two days of intensive work.

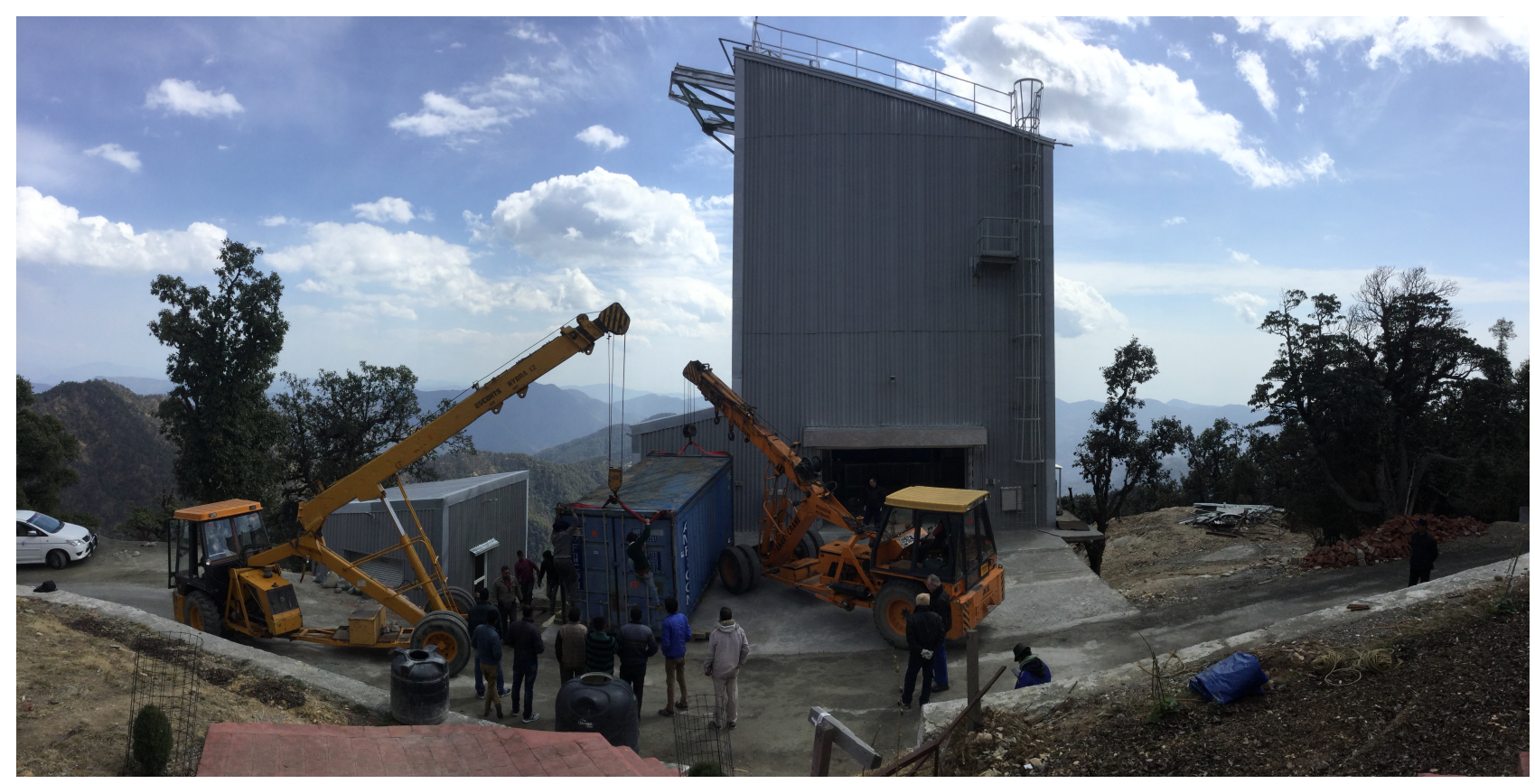

Figure 7: Big blue container with the ILMT equipment just installed in front of the compressor and ILMT buildings.

During the period 4-10 March 2017, the AMOS, ARIES and Liège University teams have contributed to the erection of the ILMT structure. Figures 8-13 illustrate the progressive installation of the four inferior pillars, the lateral reinforcing bars, the four superior pillars and the spider structure to hold the optical corrector. We may also see the presence of a deployable platform to access the prime focus of the telescope. After unpacking the optical corrector (see Fig. 14), the latter has been assembled with a tip-tilt and focus adjusting device. This whole system has subsequently been mounted on 
the structure of the prime focus holder (see Fig. 15).

The AMOS team members have then precisely positioned on 16 March 2017 the air bearing just below the center of the optical corrector. The air bearing has then been chemically and firmly anchored inside the concrete of the central pier (see Fig. 16). It has been adjusted by means of three screws belonging to the three-point mount that aligns the axis of rotation parallel to the gravitational field of the Earth. On 17 March 2017, the mirror has been set on the air bearing (see Fig. 17). This light and rigid mirror consists of a foam core sandwiched between carbon fiber sheets. Two layers of polyurethane have subsequently been cast while the recipient was spinning around its vertical axis. Its shape is thus that of a paraboloid with a focal length near $8 \mathrm{~m}$. The construction of this mirror took place at AMOS in 2009.

A nice view of the whole ILMT can be seen in Figs. 18 and 19. The first photograph was taken from the top of the roof of the ILMT building. We clearly see the optical corrector projected on the primary mirror. An interface between the corrector and the CCD camera still needs to be installed as well as the CCD camera itself. Let us also note that during observations with a liquid mirror, no mercury vapors are generated after some time (typically 8 hours) because the surface of mercury gets covered with a thin layer of mercury oxide that prevents any further evaporation. Finally, Fig. 20 shows a view on the compressor (front), the control (middle) and the main ILMT (back) buildings rather than Figs. 21-24 present aerial views of the ARIES Devasthal Observatory around the ILMT site.

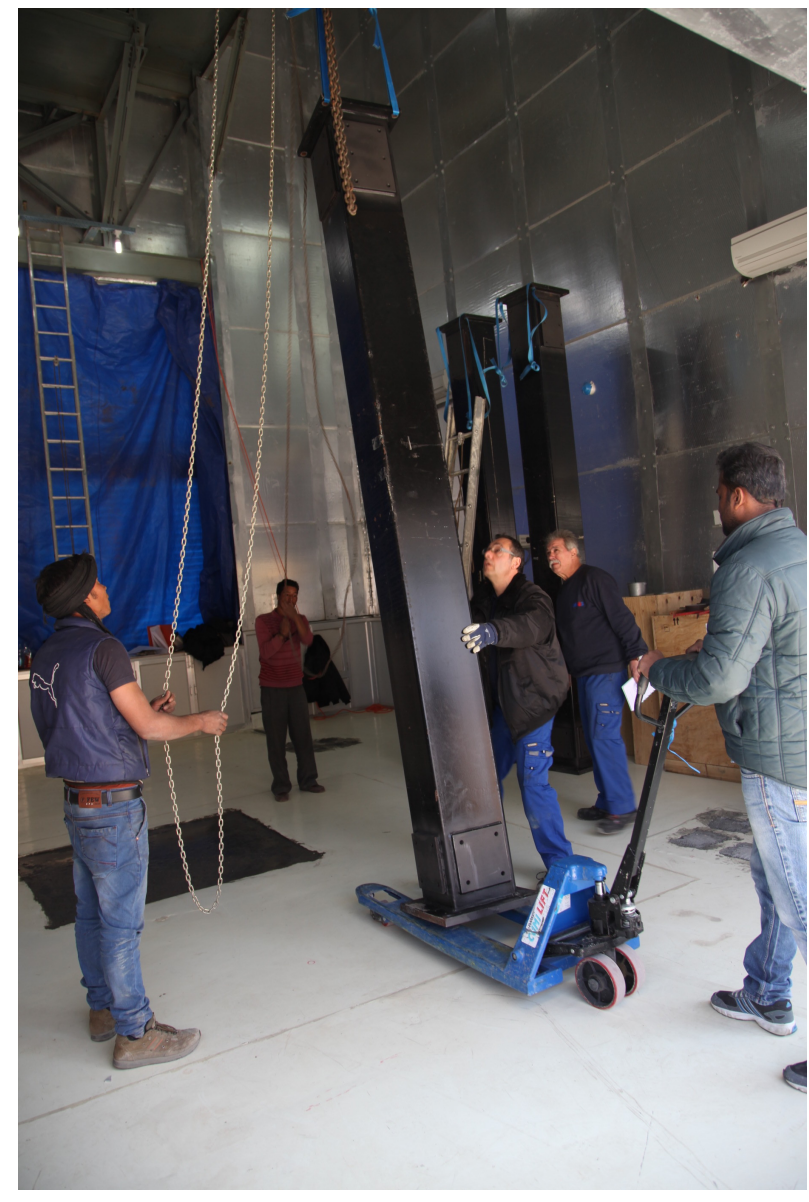

Figure 8: Erection of the ILMT structure on $4 / 3 / 2017$.

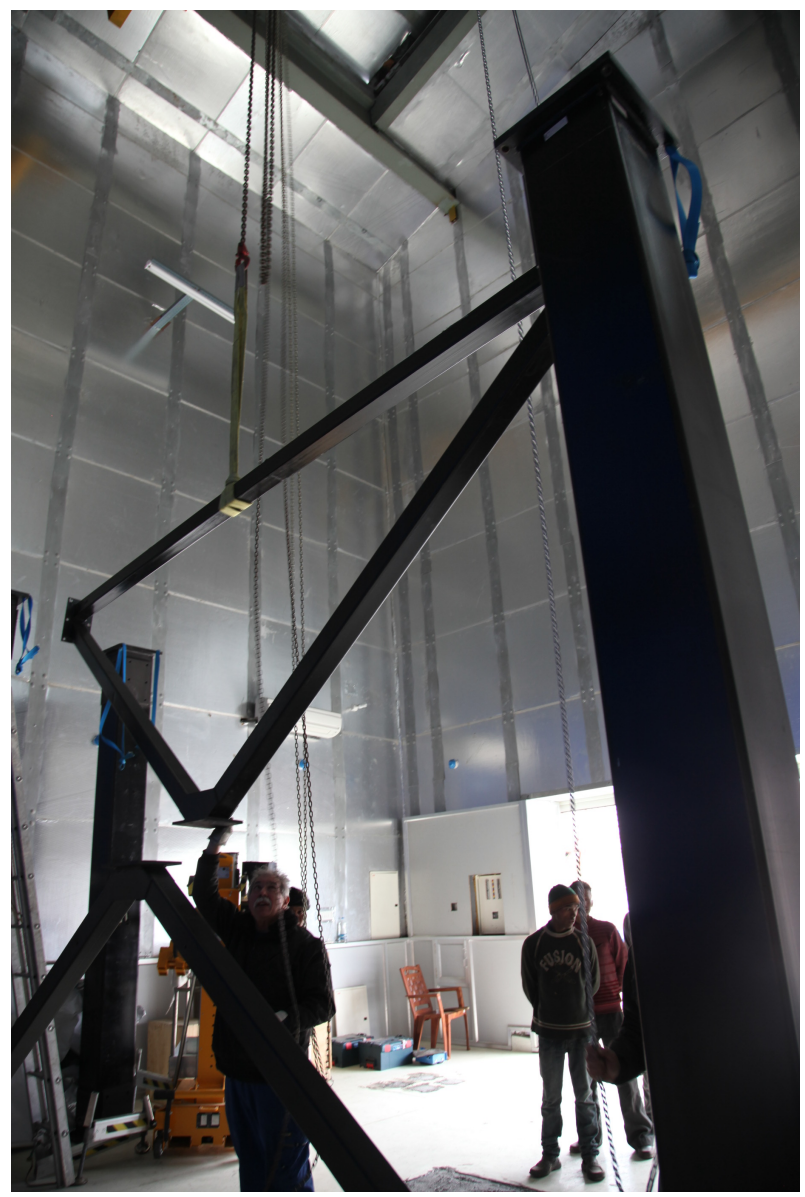

Figure 9: Erection of the ILMT structure on 5/3/2017. 


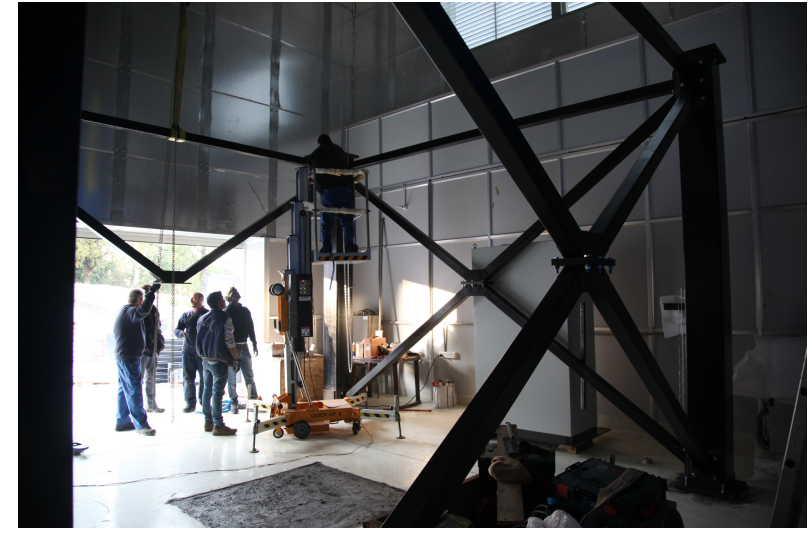

Figure 10: Erection of the ILMT structure on 5/3/2017.

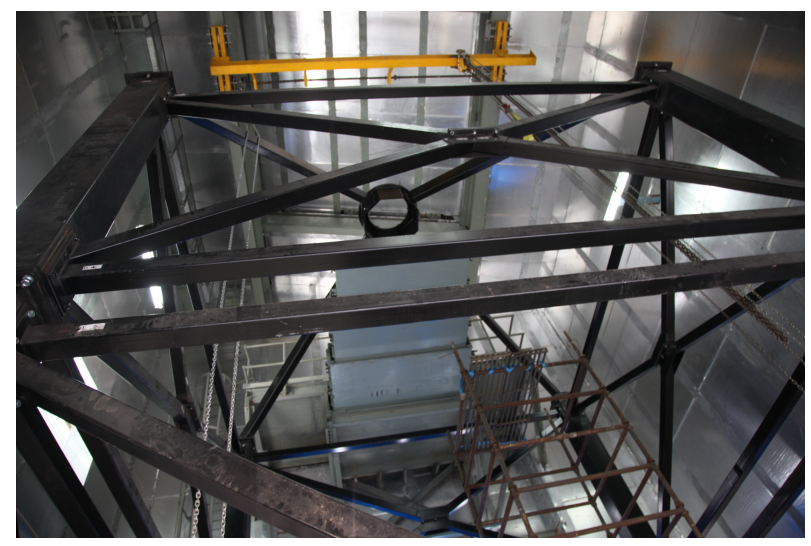

Figure 12: Erection of the ILMT structure on 9/3/2017. The platform to access the prime focus is being unfolded.

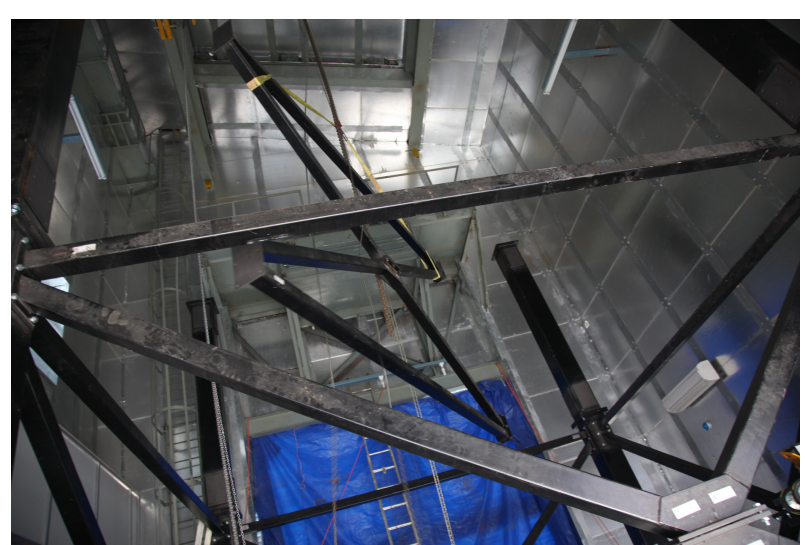

Figure 11: Erection of the ILMT structure on $7 / 3 / 2017$.

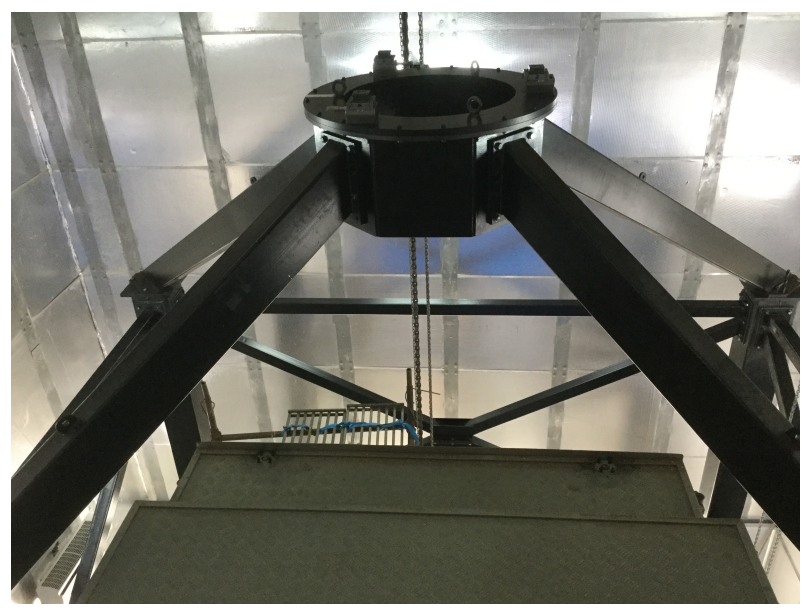

Figure 13: The central structure to hold the optical corrector on 10/3/2017.

\section{Expected performances of the ILMT over those of a conven- tional telescope}

Given the zenith observing mode of a liquid mirror telescope and in order to access the northern galactic pole, the Devasthal observatory is ideally located in latitude (near $\left.+29^{\circ} 22^{\prime} 26^{\prime \prime}\right)$. From this site, a deep $\left(i^{\prime}=22\right.$ mag.) survey will approximately cover 90 square degrees at high galactic latitude, which is very useful for gravitational lensing studies as well as for the identification of various classes of interesting extragalactic objects (cf. supernovae, clusters, etc.). Preliminary estimates of the photometric and astrometric accuracies achievable with the ILMT as a function of the magnitude of point-like objects may be found in the PhD thesis of Brajesh Kumar (2014, unpublished but accessible via the URL: http://orbi.ulg.ac.be/handle/2268/174851).

Liquid mirror telescopes (hereafter LMTs) cannot be tilted and hence cannot track the way conventional telescopes do. As written above, to track with imagery, one relies on the TDI technique, also known as drift scan, that uses a CCD detector that tracks by electronically stepping its pixels. The information is stored on disk and the night observations can be co-added with a computer, resulting into longer integration time images. Conversely, the image resulting from the subtraction of a nightly recorded image by a reference image characterized by a high $\mathrm{S} / \mathrm{N}$ may easily lead to the identification of photometrically and/or astrometrically variable objects, including variable point-like components 


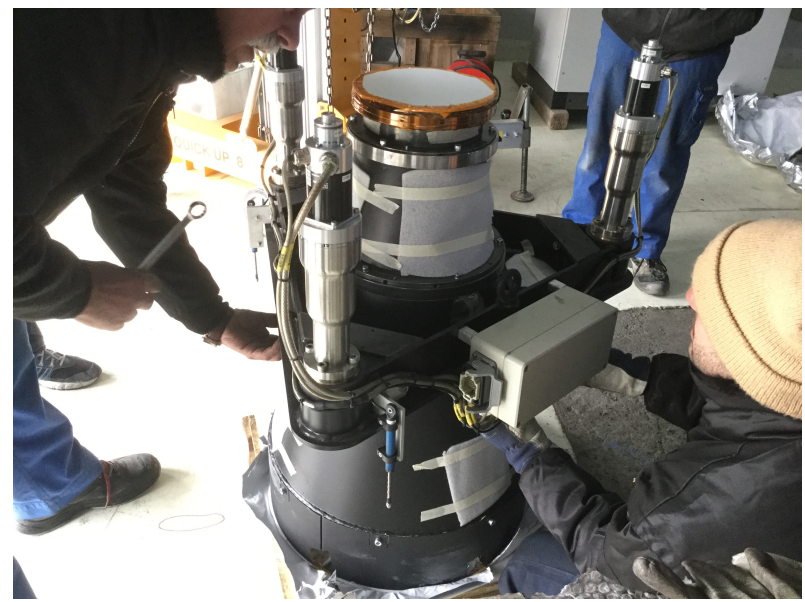

Figure 14: The optical corrector being unpacked on 11/3/2017.

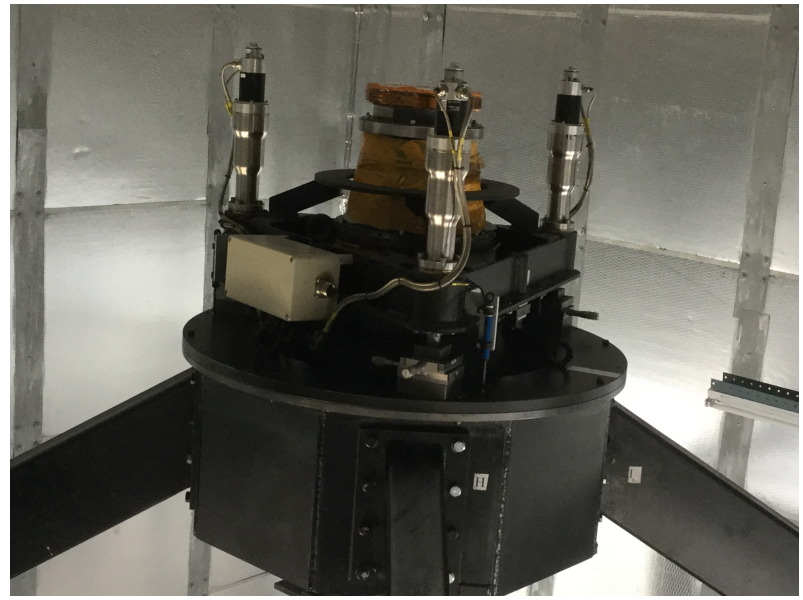

Figure 15: The optical corrector, assembled with the tip-tilt and focus adjusting devices, has been lifted and mounted on the prime focus holder.

superimposed on extended objects (cf. multiply imaged quasars, supernovae, etc.). The collected data will be ideally suited to perform a deep photometric and astrometric variability survey over a period of typically 5 years. A pipeline to digest all those data is being set at the Poznań Observatory.

Some research projects (e.g. time consuming surveys, long term photometric monitoring programs) simply cannot be envisioned with classical telescopes but become possible with a dedicated liquid mirror telescope. This is particularly true for the types of research where the specific region of the observed sky is not very important (e.g. cosmology). Furthermore, the quality of the recorded observations is optimal at zenith since both the seeing and the transparency are the best there, at all times. Moreover, the observing efficiency is very high. Indeed, when observing each night the same strip of sky, there is no time lost for slewing, field acquisition, readout times, etc.

Let us finally note that flat fielding and CCD image de-fringing are much more accurate than during classical observations since the images are actually formed by averaging the signal over entire CCD columns (in the direction of the scan). Among the apparent disadvantages, let us recall that one can only observe at zenith a strip of constant declination. At the latitude of $+29^{\circ} 22^{\prime} 26^{\prime \prime}$, a band of approximately half a degree covers 156 square degrees, with 88 square degrees being covered at high galactic latitude $\left(|b I I|>30^{\circ}\right)$. The nightly integration times are rather short, typically $\sim 100 \mathrm{sec}$. but as already stated before it is always possible to co-add data from selected nights in order to get longer integration times. The volume of collected data is estimated to be $\sim 10$ Gby per night.

\section{Scientific cases}

The advantages and limitations of this unusual type of telescope constrain the science that is feasible and thus the science requirements. The ILMT will be entirely dedicated to projects of high scientific interest dealing with astrometric or photometric variability. They concern galactic and extragalactic astrophysics. A short list of the science drivers namely includes:

i) the statistical determination of the cosmological parameters $H_{0}, \Omega_{M}$ and $\Omega_{\Lambda}$ based upon surveys for multiply imaged quasars which consist of compact gravitational lens systems, ii) the statistical determination of these same cosmological parameters based upon surveys for supernovae, iii) a search for quasars and observational studies of large scale structures, iv) the determination of trigonometric parallaxes of faint nearby objects (e.g. faint red, white, brown dwarfs, halo stars and other very low 


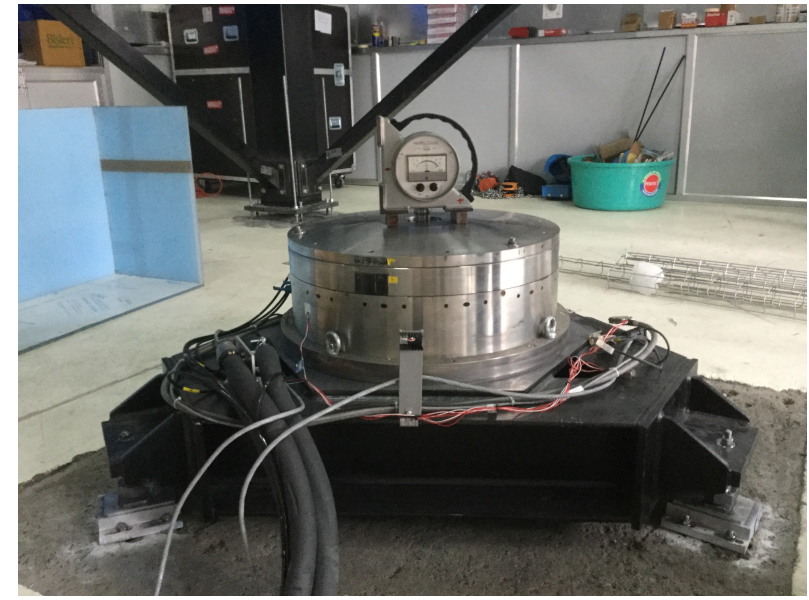

Figure 16: Positioning and centering of the air bearing just below the optical corrector. The air-bearing has been adjusted horizontally by means of three screws grounded to the floor.

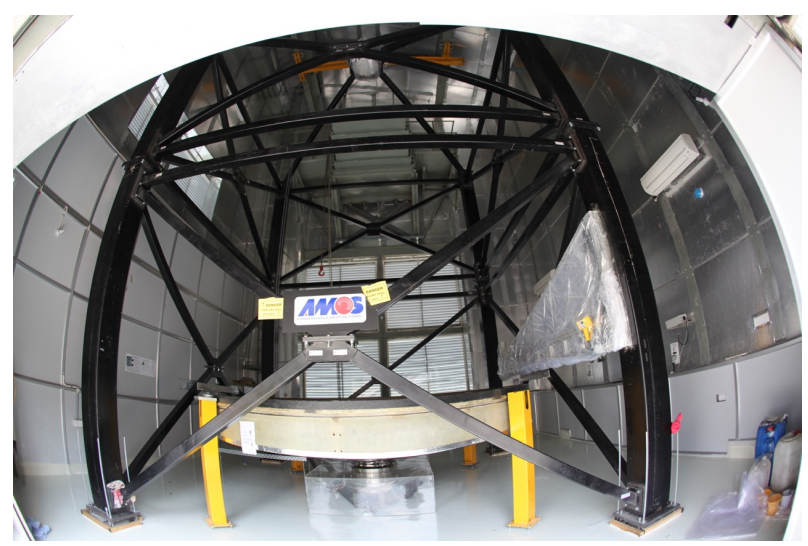

Figure 17: Fish eye view of the ILMT mirror, the air bearing and four safety yellow pillars to prevent any switchover of the mercury bowl.

mass stars, etc.), v) the detection of high stellar proper motions to probe a new range of small scale kinematics (stars, trans-neptunian objects, etc.), vi) a wide range of photometric variability studies (cf. photometry of stars, RR Lyrae, transiting extra-solar planets, novae, supernovae, micro-lensing and other transient events, photometry of variable AGN over day to year time scales, etc.), vii) the detection of low surface brightness and star-forming galaxies, and other faint extended objects (galactic nebulae, supernova remnants, etc.), viii) serendipitous phenomena, and finally, ix) the production of a unique database for follow up studies with the $3.6 \mathrm{~m}$ Devasthal Optical Telescope (DOT). Follow up studies with other large telescopes (cf. VLT, Gemini, Keck, GranTecan, SALT, etc.) will also be encouraged.

More details on the 4-m ILMT project and its scientific cases may be found in Claeskens, Jean \& Surdej (2001), Jean, Claeskens \& Surdej (2001), Surdej et al. (2006), and via the following web site: http://www.ilmt.ulg.ac.be

\section{Conclusions}

For zenith-pointing observations, LMTs can deliver the same performance as classical telescopes, with much lower cost and greater simplicity of operation. Although several liquid mirror telescopes (e.g., the 2.7-m and the 6-m University of British Columbia and NODO LMTs) have been previously used for sky observations (cf. space debris, earth atmosphere, sodium atmospheric layer), they were first-generation instruments not optimized for astronomy, nor located at high-quality astronomical sites (Borra, Hickson \& Surdej, 2009). The ILMT has essentially been developed for celestial observations from a good astronomical site. The 4-m ILMT is an instrument that will be entirely dedicated to a photometric and astrometric variability survey of a narrow strip of sky ( $\sim$ half a degree) passing through the zenith. Each night, a long CCD image recorded under the best image conditions (atmospheric seeing and extinction) will be compared to a reference one and any transient source or highly variable object should be easily detected. Thanks to the proximity of the $3.6 \mathrm{~m}$ DOT, follow-up strategies should be easily implemented. We expect to get first light after the monsoon of 2018. 


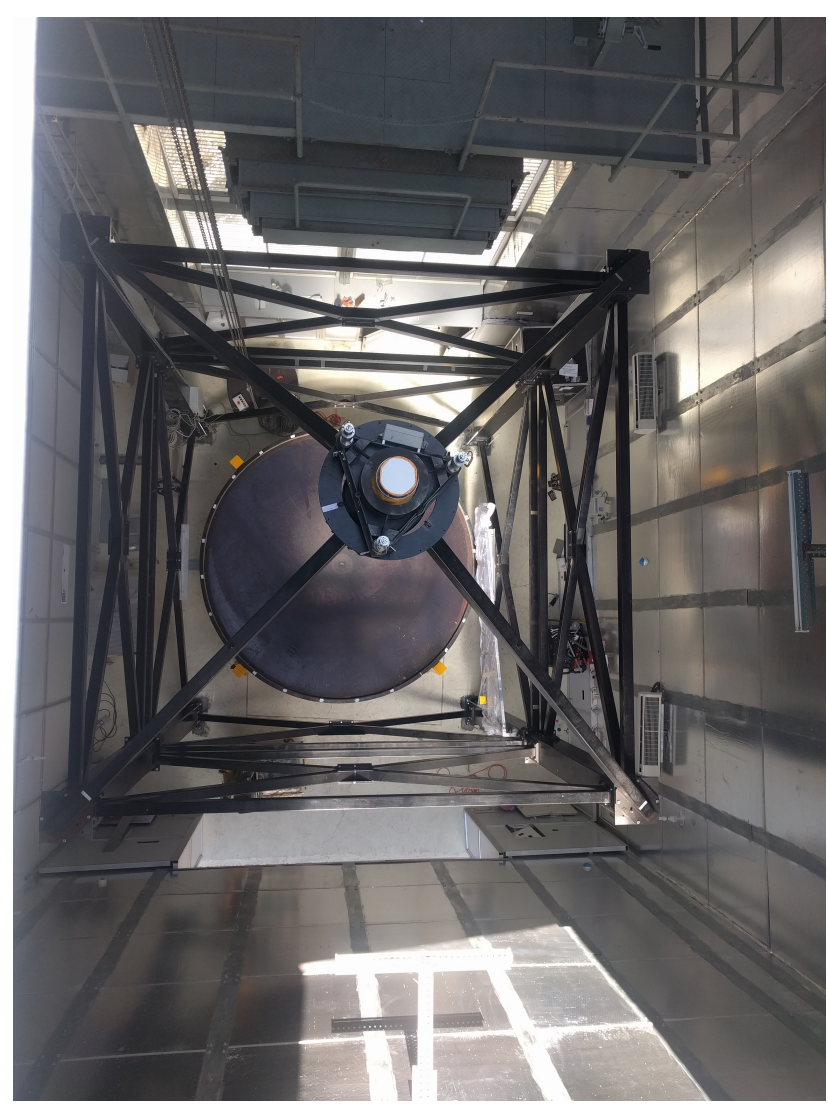

Figure 18: View of the ILMT from the top of the roof. The optical corrector is superimposed over the mirror. On the top is visible the folding platform.

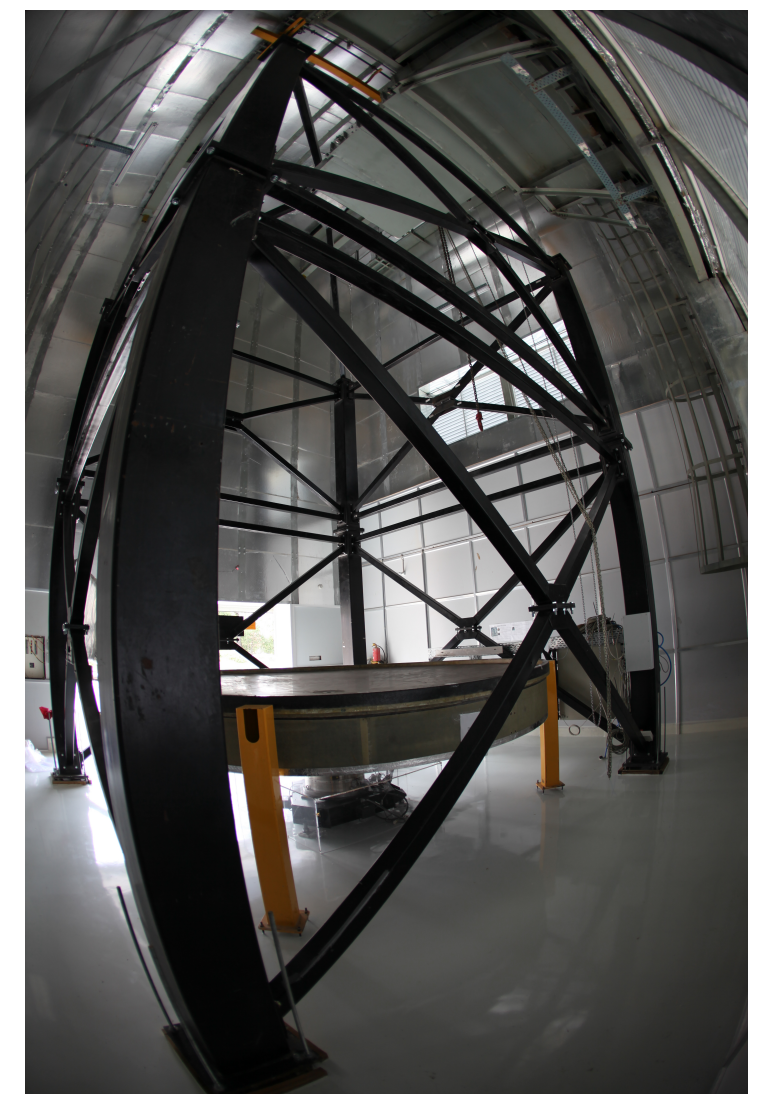

Figure 19: Fish eye view of the ILMT from the rear side wall of the main building

\section{Acknowledgements}

This research has been supported by Région Wallonne (Belgium) under the convention 516238, F.R.S.-FNRS (Belgium), the Liège University and the Natural Sciences and Engineering Research Council of Canada. J.S. and J.P.S. wish to express their special thanks to Ir. Alain Gillin, Director, for his comprehension in renewing the Convention $n^{\circ} 516238$ 'TML4M' during many years and Prof. Govind Swarup, Chair of the ARIES Governing Council, for his constant and very important support. The authors wish to thank BINA for financial support and the good organization of this workshop.

\section{References}

Borra E. F., Content, R., Girard L. 1993, ApJ 418, 943

Borra E., Hickson P., Surdej J. 2009, OptPN, 20, 28

Claeskens J.-F., Jean C., Surdej J. 2001, ASP Conference Series, 239, 373

Hickson P., Pfrommer T., Cabanac R. et al., 2007, Pub. Astron. Soc. Pacific, 119, 444

Hickson P., Racine R. 2007, Pub. Astron. Soc. Pacific, 119, 456

Jean C., Claeskens J.-F., Surdej, J. 2001, ASP Conference Series, 237, 423

Mailly E., 1872, De l'astronomie dans l'Académie royale de Belgique. Rapport séculaire (1772-1872), 26

Pfrommer T., Hickson P., She C.-Y. 2009, Geophys. Res. Lett., 36, L15831

Pfrommer T., Hickson P. 2010, JOSA A, 27, A97

Pfrommer T., Hickson P. 2014, A\&A, 565, 102

Surdej J., Absil O., Bartczak P. et al. 2006, Proceedings of the SPIE conference, Orlando, Vol. 6267, 626704 


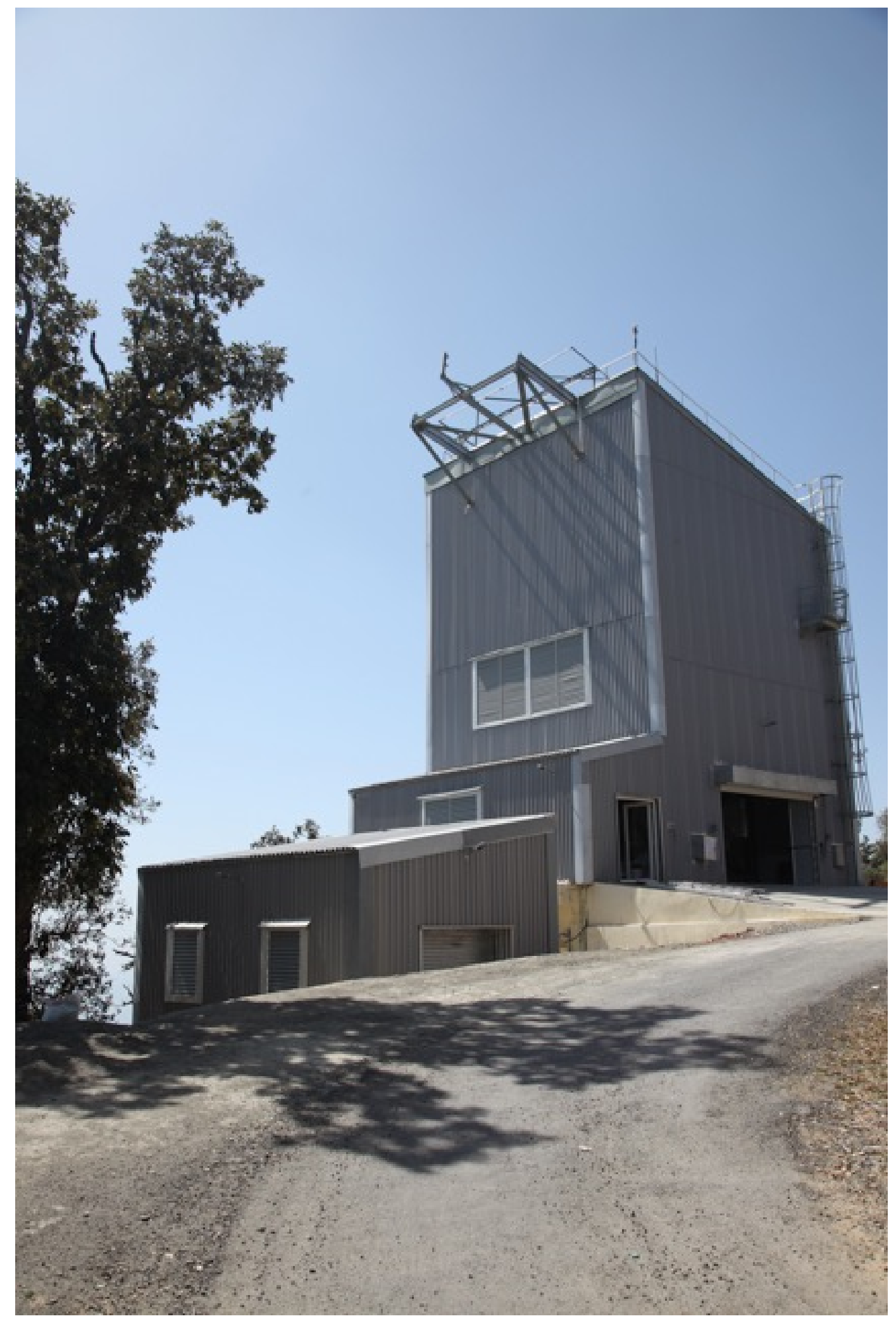

Figure 20: View of the ILMT compressor, control and main ILMT buildings. 


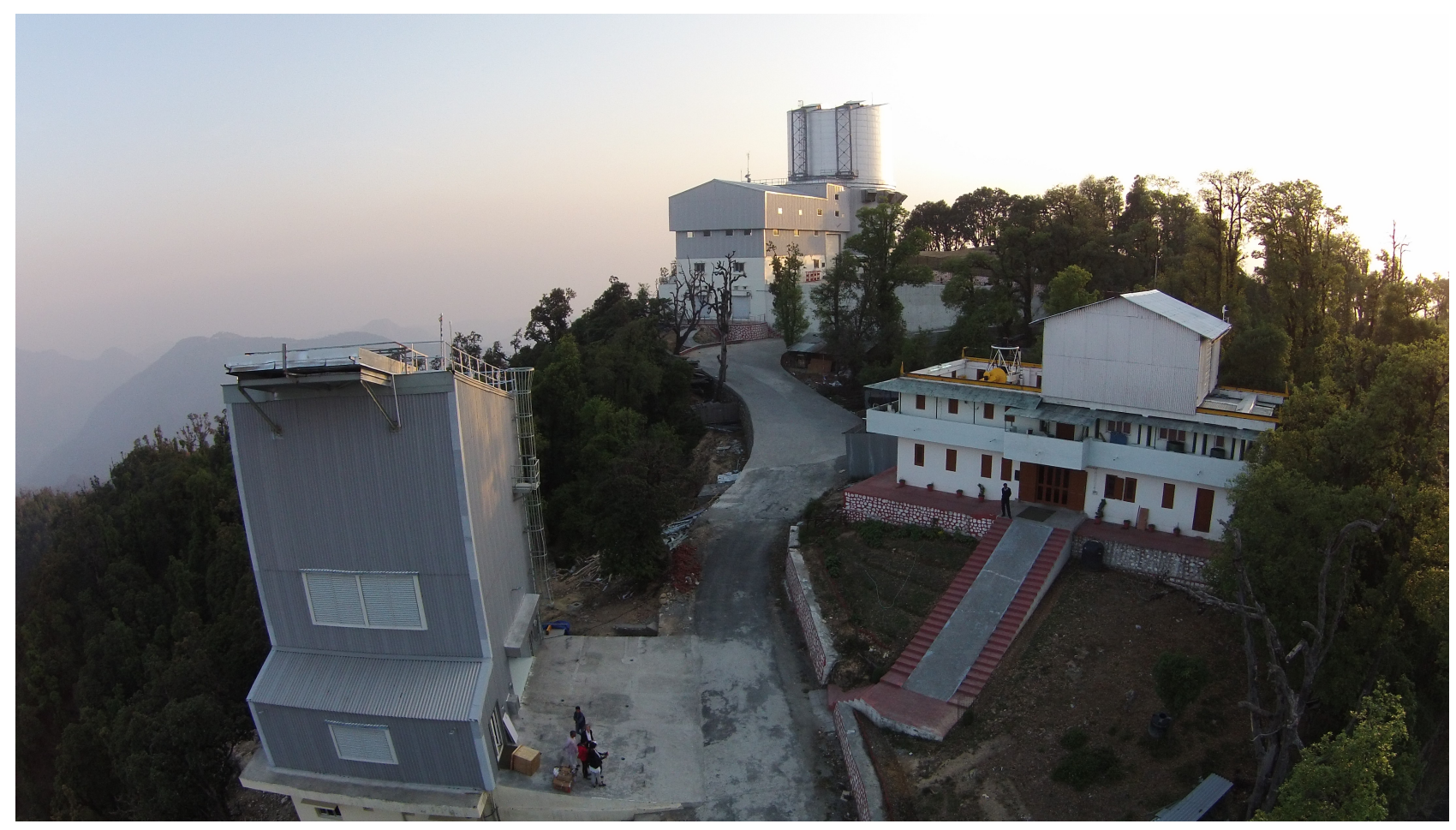

Figure 21: Aerial view of the ILMT control and main ILMT buildings on the left, the $3.6 \mathrm{~m}$ DOT in the middle top and the $1.3 \mathrm{~m}$ telescope on the right.

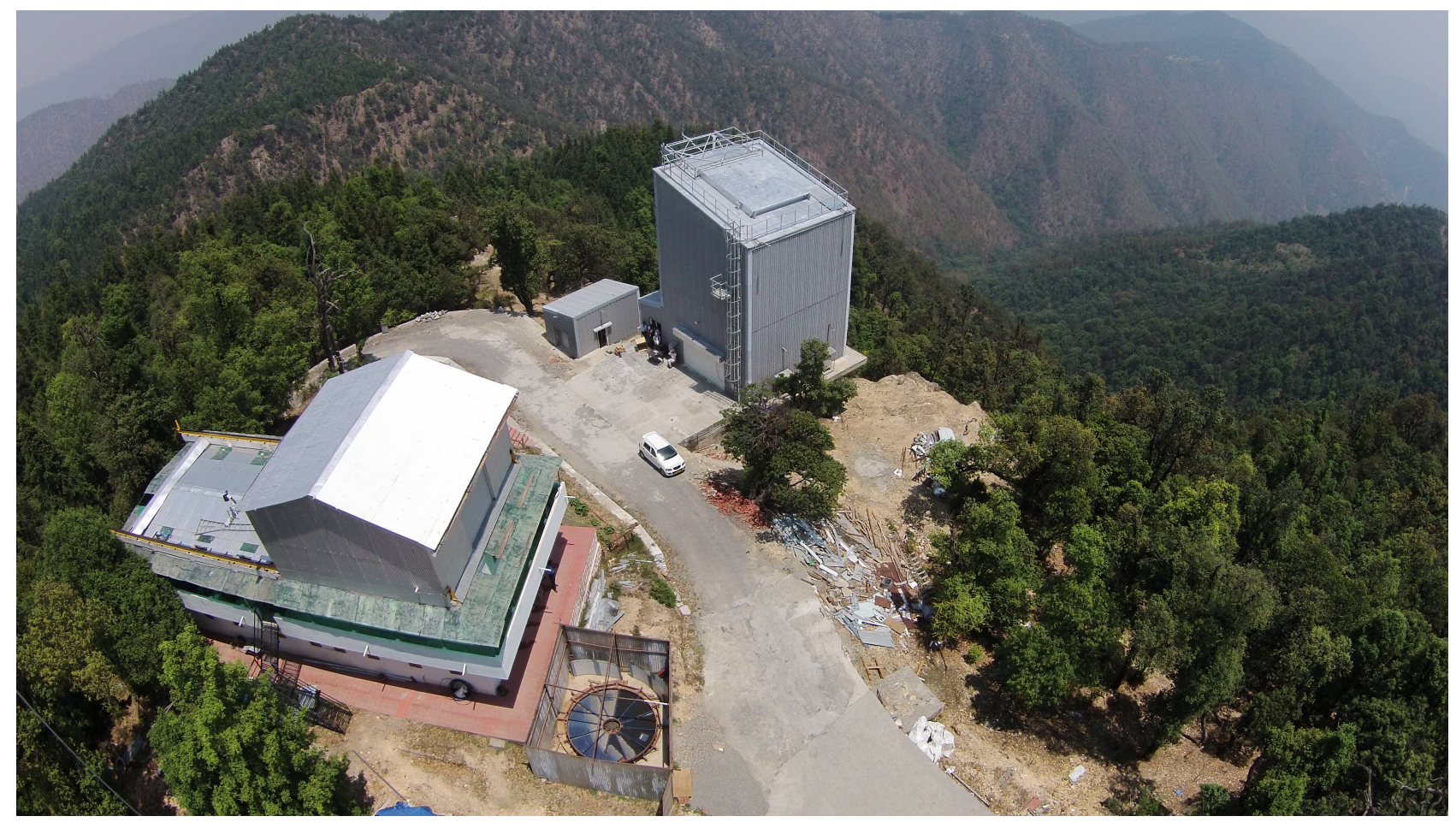

Figure 22: Aerial view of the ILMT (right) and 1.3m telescope (left) buildings. The support structure of the 4-m ILMT bowl is seen near the middle bottom of the picture. 
Bulletin de la Société Royale des Sciences de Liège, Vol. 87, Actes de colloques, 2018, p. 68 - 79

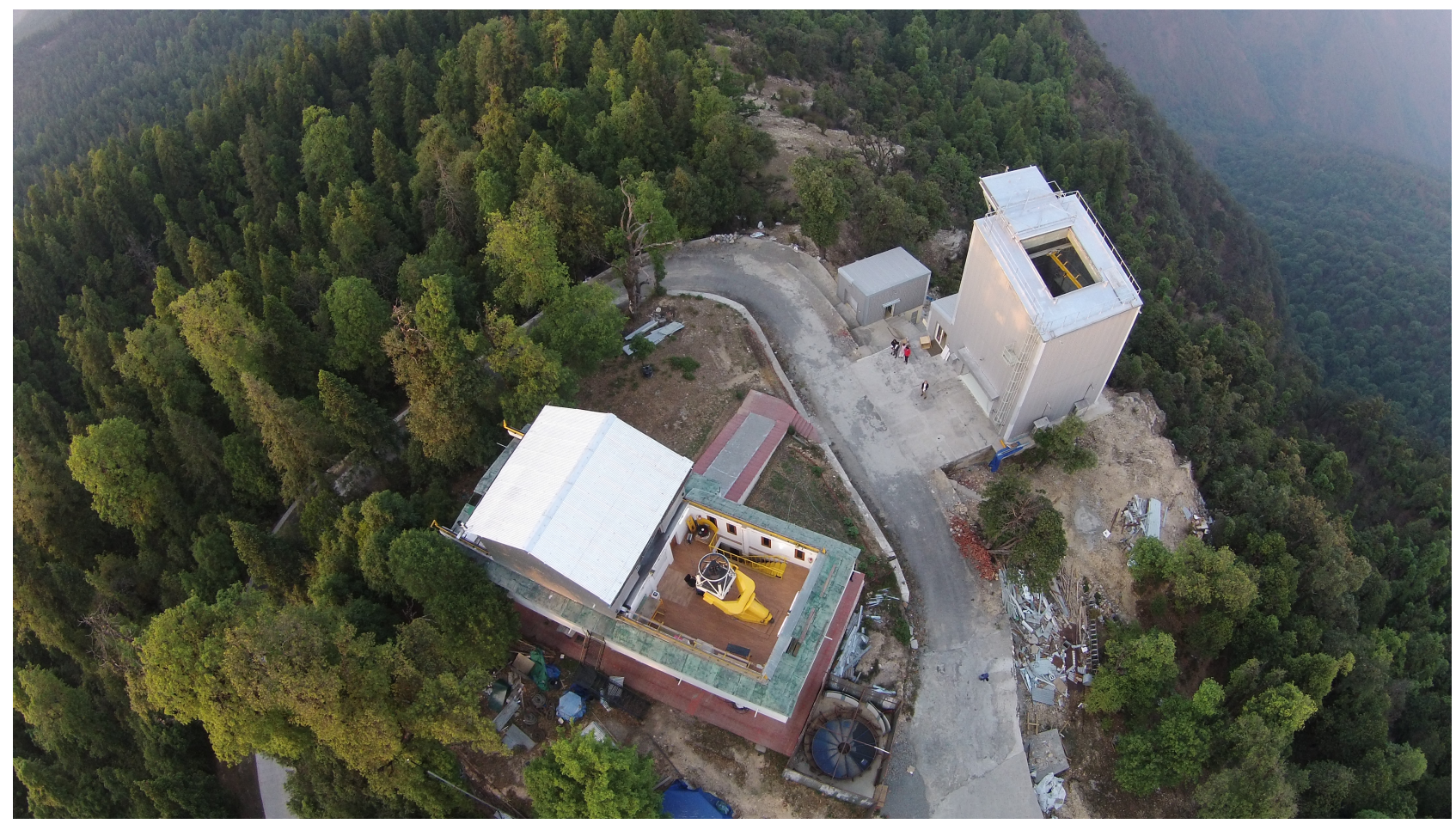

Figure 23: Aerial view of the ILMT building with its roof open (right) and the $1.3 \mathrm{~m}$ telescope (left).

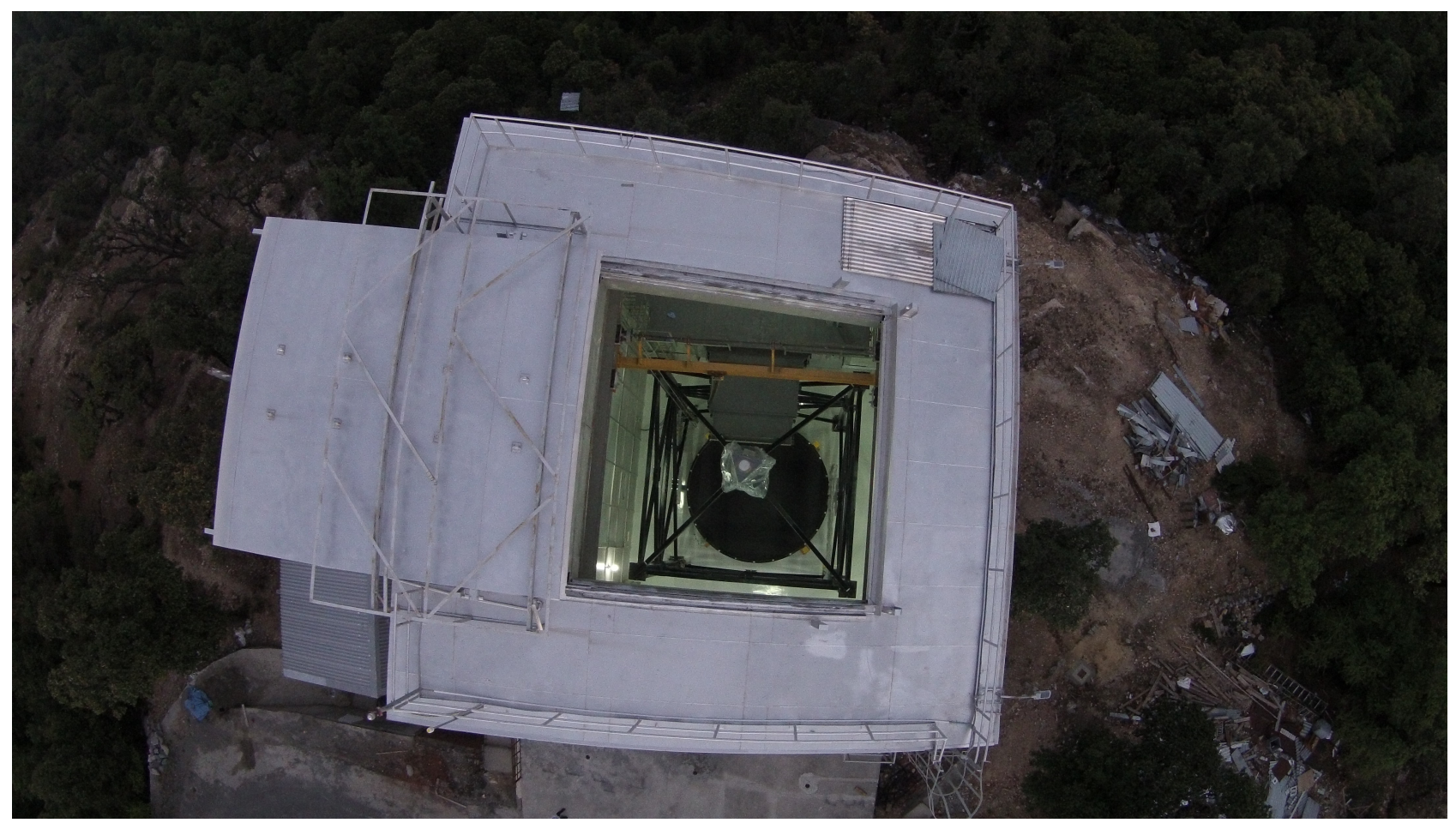

Figure 24: Aerial top view of the ILMT. 